\title{
A Hybrid Model for a Hybrid Science
}

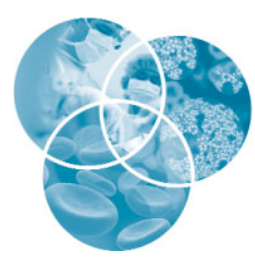

$\mathrm{T}$ Those of you who know me personally will be surprised to see me on this masthead, as I have said that I would not edit a 'nanomedicine journal.' Despite initial appearances, I hope to convince you that this publication is something different: a living document, a hybrid that recognizes the diversity of the research that contributes to our field.

I have several concerns about the usefulness of current journal formats for nanomedicine and nanobiotechnology. First, I feel that, because this is such a cross-disciplinary field, traditional journals often are not able to serve their readers effectively. By necessity, most journals tend to be put together from a single perspective, be it medical, nanomaterials, or nanodevices, that does not address the needs of all interested readers. Second, I am concerned that the standard journal format does not offer an appropriate entree to students and professionals attempting to enter the field, again from a variety of disciplines. This is because most journals publish research or review papers that are highly technical in nature, and such articles do not provide the type of perspective that a student, or a professional in an adjacent area of research, needs to help them find their place in a field. In contrast, a textbook for this rapidly evolving field would be extremely difficult to keep current.

So, if not a standard journal, what kind of publication is Wiley Interdisciplinary Reviews: Nanomedicine and Nanobiotechnology? This will be a unique endeavor that will combine the best features of online reference works (it is a comprehensive, authoritative, frequently updated resource comprising invited contributions from leading researchers) and review journals (it offers high online visibility as well as journal-type abstracting and indexing that will confer appropriate professional credit on authors). This will be a truly living document that will exist primarily in electronic format but can also be produced in print versions periodically to provide access to those without the skill or inclination to use electronic references. Most importantly, this work is meant to provide an ongoing perspective on the development of the field. Several distinctive article types have been commissioned. Advanced reviews will survey specific areas in a citation-rich format suitable for graduate students and researchers. Opinions on issues related to nanomedicine and nanobiotechnology will be invited from thought leaders in the field. Overviews will allow people not entirely familiar with an area to rapidly gain a perspective on what might be important, new or innovative. Finally, we will include shorter contributions, known here as Focus articles, in which authors will offer perspectives on their own work in the field. This will provide them with an opportunity to place what they are doing in the context of the long-term development of nanomedicine and nanobiotechnology.

One of the key benefits of this publication is that it will be written from multiple viewpoints ranging from medical to material science. Importantly, we hope to make this content accessible to individuals in diverse disciplines in such a way as to provide a single source and platform to discuss and define the field. We will develop an interactive online forum that will allow editors and contributors to discuss published articles and offer input to the evolving knowledge base. In addition, the highly structured format of the publication will allow instructors to create class texts for different disciplines (such as medicine or bioengineering or material science) from the same set of articles. This will provide the novice with a means to enter the field and gain familiarity with many aspects that would have previously required diverse compilations of single articles from different sources.

Thus, I believe this evolving publication will not only foster new initiatives in nanomedicine and nanobiotechnology but will also bring new investigators to the field. As with any new endeavor, I am sure there are going to be growing pains, and it will take an ongoing effort to try and achieve our goals. Wiley-Blackwell is firmly committed to this concept and believes it will fundamentally change the separation between online journals and reference works. We look forward to your feedback and hope that you find this new publication of interest, and stay with us to see how it evolves.

Jim Baker

Director of Michigan Nanotechnology

Institute for Medicine and the

Biological Sciences Chief

University of Michigan

Ann Arbor, MI 